\title{
Poly(D,L-lactic-co-glycolic acid) Nanoencapsulation Reduces Erlotinib-Induced Subacute Toxicity in Rat
}

\author{
Marslin Gregory ${ }^{1,3}$, Caroline J. Sheeba ${ }^{2}$, V. K. Kalaichelvan ${ }^{1}$, R. Manavalan ${ }^{1}$, \\ P. Neelakanta Reddy ${ }^{3}$, and G. Franklin ${ }^{4}$ ** \\ ${ }^{1}$ Department of Pharmacy, Annamalai University, Chidambaram, India \\ ${ }^{2}$ Life and Health Sciences Research Institute (ICVS), University of Minho, Portugal \\ ${ }^{3}$ Department of Bioorganic Chemistry and Toxicology, Central Leather Research Institute, Chennai, India \\ ${ }^{4}$ Centre for the Research and Technology of Agro-Environment and Biological Sciences (CITAB), \\ Department of Biology, University of Minho, Portugal
}

\begin{abstract}
Erlotinib- $\mathrm{HCl}$ is a quinazoline derivative used as a drug in the therapy of non-small-cell lung cancer. The present study was conducted to compare the subacute toxicity induced by Erlotinib- $\mathrm{HCl}$ delivered to rats as nanoparticles and as free drug. Wistar rats were orally administered with a daily dosage of $200 \mathrm{mg} \mathrm{kg}^{-1}$ Erlotinib-HCl either as free drug or as Poly(D,L-lactic-co-glycolic acid) (PLGA) encapsulated nanoparticles. After four weeks of treatment, the animals were analyzed for toxicological changes. Although nanoparticulate form of the drug did not induce any toxicity, free drug significantly reduced the levels of white blood cells (WBC), red blood cells (RBC) and haemoglobin, while increasing the levels of neutrophils and corpuscular haemoglobin. Moreover, aspartate aminotransferase (AST) and alanine aminotransferase (ALT) levels were significantly increased in the animals administered with free drug. Histopathological studies confirmed significant damage to the internal organs of animals treated with free drug. Whereas, the internal organs of animals treated with the drug encapsulated in PLGA nanoparticles were more or less similar to the healthy organs. Our results show that Erlotinib-HCl delivered in the form of nanoparticles has less toxic effect than the free drug in experimental rats.
\end{abstract}

Keywords: Erlotinib-HCl, Non-Small-Cell Lung Cancer, PLGA Nanoparticle, Subacute Toxicity.

\section{INTRODUCTION}

Non-small-cell lung cancer accounts for approximately $75 \%$ of lung cancers and a leading cause of cancerrelated death in men and women with overall cure rates of less than $15 \% .^{1}$ Recent molecular biology studies have revealed that aggressive tumor activity of non-small-cell lung cancer is generally correlated with the over expression of the Epidermal Growth Factor Receptor (EGFR) tyrosine kinase. ${ }^{1}$ Tyrosine kinase plays a critical role in many cell signaling pathways that influence cell division, apoptosis, motility and adhesion. ${ }^{2}$ Activated forms of tyrosine kinases can enhance tumor cell proliferation and growth, induce antiapoptotic effects and promote angiogenesis and metastasis. Erlotinib- $\mathrm{HCl}$ specifically inhibits tyrosine kinase receptors from binding to their legends, thus blocks the downstream signaling pathways and tumor activity. Hence, this compound is currently used in the therapy of non-small-cell lung cancer. ${ }^{3,4}$

${ }^{*}$ Author to whom correspondence should be addressed.
Erlotinib- $\mathrm{HCl}$ is available in the market as film coated tablet for oral administration. This route is the most preferred and easy way of drug delivery. However, the drug administered through this route is poorly available mainly because of the biopharmaceutical (poor solubility, poor permeability and/or instability in gastrointestinal environment) and pharmacokinetic (extensive first pass metabolism and/or rapid clearance) hurdles. ${ }^{5}$ Moreover, cancer treatments normally require long term drug administration and the drugs generally attack both normal cells and cancer cells, when the drug is given as an injection or in tablet form for a long time. ${ }^{6}$ As the aforementioned limitations are unavoidable in oral administration, methods to protect the drug from external harsh conditions (chemical and enzymatic degradation) of gastro intestinal track and sustained release are necessary.

Delivery of anticancer agents as nanoparticles has received much attention due to the unique accumulation behavior of nanoparticles at the tumor site. ${ }^{7-9}$ In the nanoparticulate drug delivery, the therapeutic agent 
of interest is encapsulated within the polymer matrix of biodegradable particles to achieve extended release. ${ }^{10,11}$ When encapsulated within a biodegradable polymer, the drug is released slowly over a period of time as the polymer degrades. Poly(D,L-lactic-co-glycolic acid) (PLGA) polymer used in this study is regarded as highly safe because it is completely metabolized by the body and approval by the FDA. ${ }^{12}$ The final degradation products such as lactic and glycolic acids of this polymer are also completely safe, as they are either excreted by the kidneys or enter the Krebs' cycle and eventually eliminated as carbon dioxide and water. ${ }^{13,14}$

From the literature it is clear that drugs encapsulated in PLGA nanoparticles exhibits reduced toxicity to normal cells/tissues compared to the free drug. ${ }^{15,16}$ Hence, Erlotinib- $\mathrm{HCl}$ was encapsulated in PLGA nanoparticles and the subacute toxicity in healthy rats was assessed and compared with the free drug administrated animals in the current investigation.

\section{MATERIALS AND METHODS}

\subsection{Drug and Chemicals}

Erlotinib-HCl was obtained from Ranbaxy Pharmaceuticals (Delhi, India). PLGA with a lactide/glycolide molar ratio of 50:50 and polyvinyl alcohol (PVA, $M W$ 22,000) were purchased from Sigma, Germany. Dichloromethane (DCM) was procured from Ranbaxy laboratory reagent (India). All reagents used in the present study were of analytical grade.

\subsection{Preparation of Erlotinib-HCl Encapsulated in PLGA Nanoparticles}

The PLGA nanoparticles with Erlotinib-HCl were prepared by sonication-solvent evaporation technique as described before. ${ }^{17}$ Briefly, an organic phase consisting of polymer (PLGA, $10 \mathrm{mg} \mathrm{mL}^{-1}$ ) and drug (Erlotinib- $\mathrm{HCl}$, $\left.0.5 \mathrm{mg} \mathrm{mL}^{-1}\right)$ dissolved in DCM $(5 \mathrm{~mL})$ was prepared. An emulsion was made by adding an aqueous solution containing a surfactant (PVA, 1\%,50 mL). This emulsion was broken down into nanodroplets by sonication $(7 \mathrm{~min})$ and the solvent was evaporated by magnetic stirring at $300 \mathrm{rpm}$ to form a colloidal suspension of PLGA nanoparticles in water. This suspension was centrifuged at $12000 \mathrm{~g}$ for $1.0 \mathrm{~h}$ to pellet down the nanoparticles. The pellet was washed three times with ultra pure water to remove any free drug.

\subsection{Characterization of the Nanoparticles}

\subsubsection{Zeta Potential}

The zeta potential of the formulations was determined by laser doppler anemometry using a Zetasizer (3000 HS,
Malvern Instruments, UK). For the measurement, $100 \mu \mathrm{L}$ nanoparticle suspension was diluted to $4 \mathrm{~mL}$ with $10 \mathrm{mM}$ $\mathrm{NaCl}$ solution and the $\mathrm{pH}$ was adjusted to 8.0 using $0.25 \mathrm{~N}$ $\mathrm{NaOH}$. An electric field of $150 \mathrm{mV}$ was applied to observe the electrophoretic velocity of the particles. All measurements were made at room temperature as described before. $^{18}$

\subsubsection{Scanning Electron Microscopy (SEM) Analysis}

Morphological characterization of nanoparticles was done by SEM analysis. Samples were prepared by dropping nanoparticle suspension onto aluminum stubs and allowing them to air-dry before coating with gold. The particles were then observed in a scanning electron microscope (JEOL JSM-6400).

\subsubsection{Determination of Drug Content of Nanoparticles}

In order to determine the dosage for administration, the drug content of prepared nanoparticles was measured. Briefly, $20 \mathrm{mg}$ nanoparticles were dissolved in $2.0 \mathrm{~mL}$ dichloromethane. Subsequently, $5.0 \mathrm{~mL}$ PBS was added. The mixture was vortexed for $1.0 \mathrm{~min}$ at $2500 \mathrm{rpm}$ and again for $2.0 \mathrm{~h}$ at $1000 \mathrm{rpm}$. After vortexing, the suspension was briefly centrifuged at $10,000 \mathrm{rpm}$ to pellet the debris. Absorbance of the supernatant was read in a spectrophotometer (Shimadzu UV-1700, India) at $348 \mathrm{~nm}$ using PBS as blank. This value was plotted on Erlotinib$\mathrm{HCl}$ standard graph to determine the drug content of the nanoformulation.

\subsubsection{Encapsulation Efficiency and Loading Capacity}

The actual loading and encapsulation efficiency of nanoparticles were determined by the following equations. Encapsulation efficiency was defined as the percentage of determined loading relative to the nominal (theoretical) loading: ${ }^{19}$

$$
\begin{aligned}
& \text { Encapsulation efficiency } \\
& =\frac{\text { The amount of drug in the nanoparticles }}{\text { Total amount of drug }} \times 100 \\
& \text { Drugloading capacity } \\
& =\frac{\text { The amount of drug in the nanoparticles }}{\text { Total amount of nanoparticles weight }} \times 100 \\
& \text { Percentage yield } \\
& =\frac{\text { The amount of recovered nanoparticles }}{\text { The amount of employedpolymer and drug }} \times 100
\end{aligned}
$$

\subsubsection{In Vitro Drug Release Measurement}

Erlotinib- $\mathrm{HCl}$ loaded in PLGA nanoparticles $(10 \mathrm{mg})$ were suspended in $2 \mathrm{~mL}$ PBS (pH 7.4) and transferred into a 
dialysis tube. The dialysis tube was then placed into a $100 \mathrm{~mL}$ bottle containing $50 \mathrm{~mL}$ PBS and the medium was stirred at $100 \mathrm{rpm}$ at $37{ }^{\circ} \mathrm{C}$. Once in every $24 \mathrm{~h}$, $1 \mathrm{~mL}$ sample was withdrawn and the accumulated drug content was measured spectrophotometrically. To compensate the withdrawn sample, $1 \mathrm{~mL}$ PBS was added after each withdrawal.

\subsubsection{Evaluation of Toxicity of Encapsulated Erlotinib-HCl in PLGA Nanoparticles}

The subacute toxicity of Erlotinib-HCl loaded PLGA nanoparticles was evaluated in six weeks old Wistar rats obtained from Government veterinary college, Chennai. Use of these animals for experimental purpose has been approved by the Animal Ethical Committee (CLRI, Chennai). All the animals were maintained in a room at $25 \pm 2{ }^{\circ} \mathrm{C}$ with 12-12 h light-dark cycle and were fed with conventional laboratory diet and unlimited supply of drinking water. They were divided into three groups (control, nano drug and free drug) each containing 5 males and 5 females for experiment. Control group received normal saline. Nano drug group received $200 \mathrm{mg} \mathrm{kg}^{-1}$ Erlotinib$\mathrm{HCl}$ encapsulated in PLGA nanoparticles (calculated based on the drug content of nanoformulation) and free drug group received $200 \mathrm{mg} \mathrm{kg} \mathrm{kg}^{-1}$ Erlotinib- $\mathrm{HCl}$ in the free form. Treatment for each group was continued for 28 days.

Animals were observed for the development of toxicological signs and symptoms such as mortality, change in body weight and feed intake throughout the study period. On day 29 after the beginning of the experiments, blood sample were collected from the orbital sinus of each animal. Blood samples for haematological and biochemical studies were collected in vials containing EDTA and in vials rinsed with heparin respectively. After blood collection, all the animals were dissected to obtain internal organs (liver, kidney, adrenal gland, testes, brain and spleen). Organs were weighed and observed for gross pathological changes. Heart, lungs, kidney and liver were preserved in $10 \%$ formalin for histopathological studies.

\subsubsection{Haematological Analyses}

Red blood cells (RBC) and white blood cells (WBC) content of the blood samples were determined in a haemocytometer. Haematocrit (HCT) and haemoglobin (Hb) contents were estimated spectrophotometrically using standard methods. Platelet (Plt) count was made using direct method and clotting time (coagulation time) by capillary method. For differential count, blood smear was stained with Leishman stain and 100 leucocytes were counted under light microscope. All other haematological parameters such as mean corpuscular volume (MCV), mean corpuscular/cell haemoglobin concentration (MCHC), lymphocytes (LYM) and neutrophils (N) were estimated in a haematology analyzer. ${ }^{20}$

\subsubsection{Plasma Biochemical Assays}

Plasma was separated from heparinised blood by centrifugation at $3000 \mathrm{rpm}$ for $30 \mathrm{~min}$ at $4{ }^{\circ} \mathrm{C}$. Total protein (TP) was estimated by biuret method. Aspartate aminotransferase (AST), alanine aminotransferase (ALT), alkaline phosphatase (ALP), total bilirubin (TB) and creatinine (CRE), were assayed in an autoanalyzer (Erba Smart Lab, India) by using diagnostic kits (Erba test kits, Transasia, India).

\subsubsection{Histopathological Examination}

Internal organs of the experimental animals were fixed in $10 \%$ formalin, embedded into paraffin and cut into $5 \mu \mathrm{m}$ thick sections in a microtome. Sections were mounted on glass slides using standard techniques. After staining with hematoxylin-eosin, the sections were examined and photographed under a light microscope equipped for photography.

\section{RESULTS}

\subsection{Characteristics of Erlotinib-HCl Encapsulated in PLGA Nanoparticles}

Scanning electron micrography of the prepared nanoparticles revealed a homogeneous solid matrix structure (Fig. 1). Particle size distribution analysis by zetasizer showed that the mean average of the prepared nanoparticles was $217 \mathrm{~nm}$. Encapsulation efficiency of the nanoparticles was found to be $22.86 \%$. The drug loading efficiency of the nanoparticles was $21.75 \%$ and the yield of nanoparticles was $42.50 \%$.

\subsection{In Vitro Drug Release from Nanoparticles}

The percentage of accumulated Erlotinib- $\mathrm{HCl}$ after different time period is shown as a graph (Fig. 2). After the initial burst release for about 4 days, the release rate of

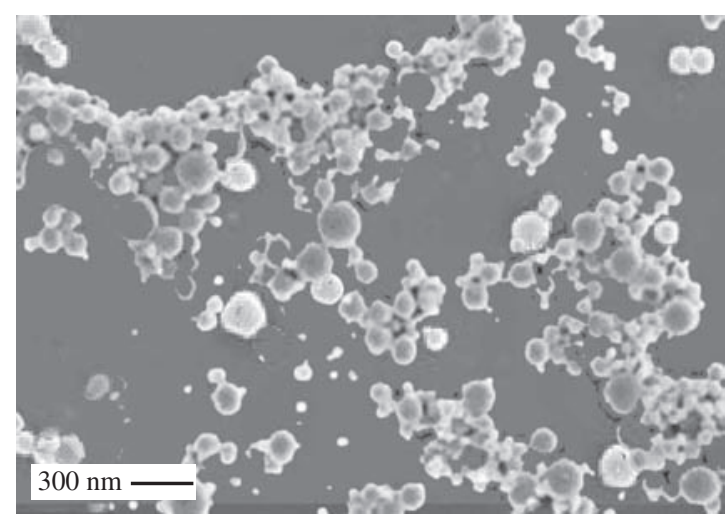

Fig. 1. Scanning electron micrograph of Erlotinib-HCl loaded in PLGA nanoparticles $($ scale bar $=300 \mathrm{~nm})$. 


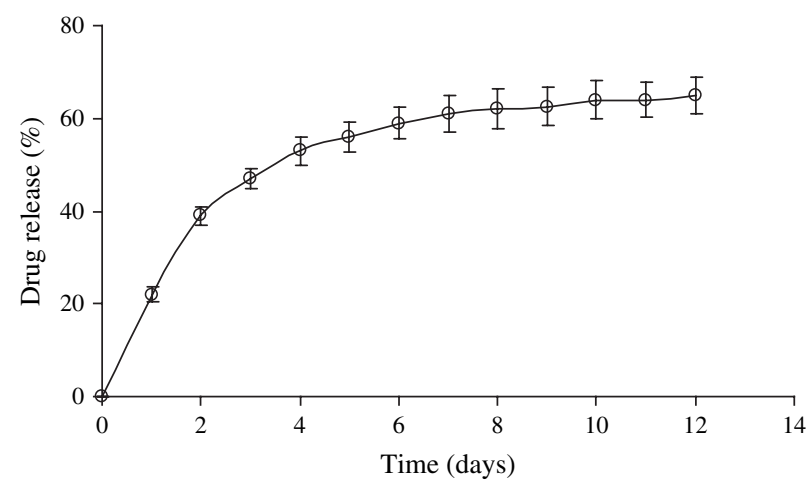

Fig. 2. The in vitro release profile of Erlotinib-HCl loaded in PLGA nanoparticles. The error bars indicate the standard deviation of the mean for 3 experiments.

Erlotinib- $\mathrm{HCl}$ from the nanoparticles slowed down. After 12 days, the amount of accumulated Erlotinib- $\mathrm{HCl}$ in PBS was calculated to be $65 \%$ of the entrapped drug.

\subsection{Changes in Body Weight}

Oral administration of Erlotinib-HCl both as nanoparticles and as free drug did not cause mortality during the experimental period. Although the growth rate of male animals was not affected (Fig. 3(a)), a reduced growth rate in female animals was observed when the free form of the drug was administered (Fig. 3(b)).

\subsection{Changes in Internal Organ Weight}

There was no change between control and nano drug groups in the weight of internal organs (liver, brain, kidneys, spleen, adrenal glands and testes). Whereas, the weight of liver and kidneys was increased significantly in free drug treated animals (both in male and female groups) over control and nano drug treated animals (Table I). Although adrenal weight was not significantly different between treatments in male groups, the weight was significantly increased in females of free drug group.

\subsection{Haematological Changes}

Haematological studies showed that there was no significant change between control and nano drug group with the exception of HCT content of female animals, which was significantly increased over control. However, significant changes (either increase or decrease) in most of the tested heamatological parameters were observed in free drug treated animals except the MCV. In addition, HCT content was not affected in male animals but it substantially reduced in female animals. There was a significant decrease in the RBC count in both male and female animals whereas the WBC count increased in the females of free drug group.
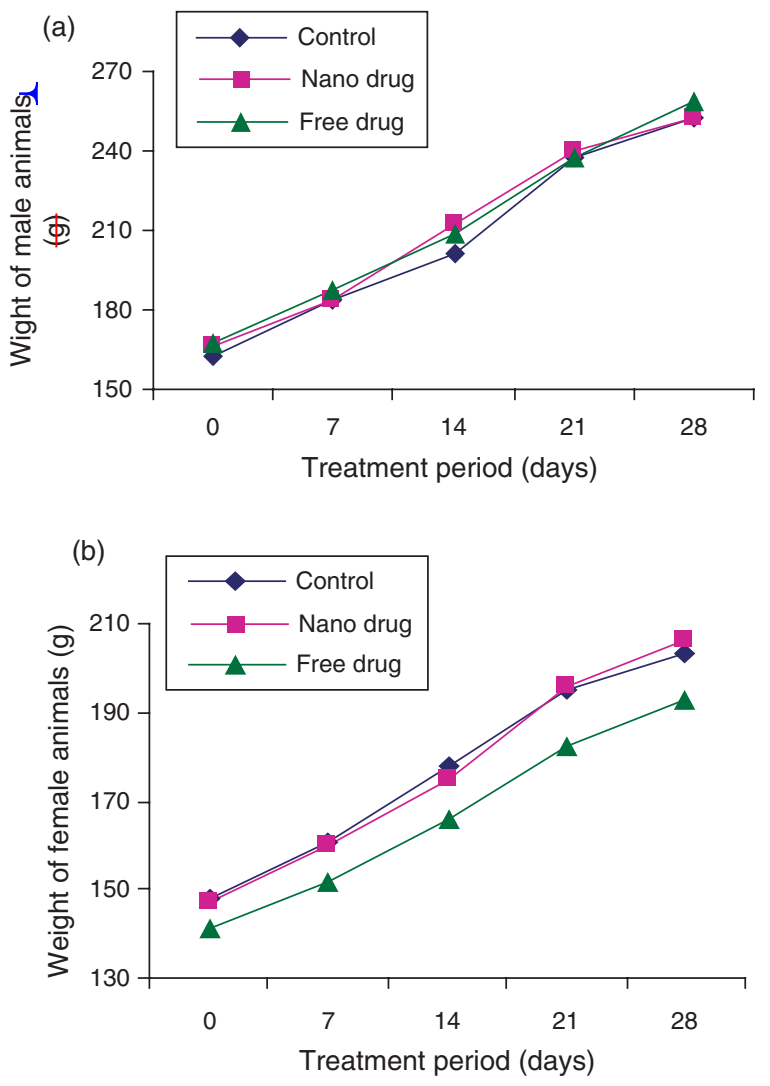

Fig. 3. Changes in the body weight of male (a) and female (b) animals during the treatment by 28 days.

\subsection{Biochemical Changes}

There was no significant change in the tested parameters between control and nano drug treated animals. Nevertheless an increased level of ALP was observed in the female animals treated with nano drug compared to control. Aspartate aminotransferase (AST), alanine aminotransferase (ALT), alkaline phosphatase (ALP), and creatinine levels were significantly increased in animals administered with free drug compared to control and nano drug groups (Table III).

\subsection{Histopathological Changes}

The histological sections of heart, lungs, kidney and liver of control animals are shown in Figures 4(a)-(d). Similar histological sections of organs of animals administered with nanoparticles with Erlotinib- $\mathrm{HCl}$ are shown in Figures 4(e)-(h). Comparison of these photomicrographs clearly shows that there were no toxicity-induced changes in the animals administered with nanoparticles with Erlotinib-HCl. However, in the organs of animals administered with free drug many toxicity related changes were observed (Figs. 4(i-1)). As seen in the figure, cardiac muscle fibres were completely damaged in the heart (Fig. 4(i)), normal architecture was lost in lung (Fig. 4(j)), 
Table I. Changes in the internal organ weight ( $\mathrm{g}$ ) of control and treated animals after 28 days of drug administration.

\begin{tabular}{|c|c|c|c|c|c|c|}
\hline & \multicolumn{3}{|c|}{ Male groups } & \multicolumn{3}{|c|}{ Female groups } \\
\hline & Control & Nano drug & Free drug & Control & Nano drug & Free drug \\
\hline Liver & $7.5 \pm 0.27$ & $7.49 \pm 0.29$ & $9.2 \pm 0.54^{a}$ & $6.46 \pm 0.50$ & $6.50 \pm 0.19$ & $8.65 \pm 0.15^{a}$ \\
\hline Brain & $1.84 \pm 0.07$ & $1.82 \pm 0.05$ & $1.79 \pm 0.04$ & $1.79 \pm 0.02$ & $1.88 \pm 0.05$ & $1.86 \pm 0.05$ \\
\hline Kidneys & $1.95 \pm 0.05$ & $1.97 \pm 0.07$ & $2.26 \pm 0.13^{a}$ & $1.54 \pm 0.06$ & $1.44 \pm 0.06$ & $2.55 \pm 0.06^{a}$ \\
\hline Spleen & $0.62 \pm 0.07$ & $0.61 \pm 0.08$ & $0.58 \pm 0.12$ & $0.76 \pm 0.13$ & $0.56 \pm 0.04$ & $0.65 \pm 0.08$ \\
\hline Adrenal & $0.05 \pm 0.01$ & $0.05 \pm 0.02$ & $0.06 \pm 0.01$ & $0.07 \pm 0.01$ & $0.071 \pm 0.01$ & $0.23 \pm 0.16^{a}$ \\
\hline Testes & $2.67 \pm 0.13$ & $2.63 \pm 0.15$ & $2.85 \pm 0.11$ & - & - & - \\
\hline
\end{tabular}

The values are expressed as mean \pm SEM of 5 animals. ${ }^{a}$ denotes significant at the level of $p<0.05$.

Table II. Haematological observation of control and treated animals after 28 days of drug administration.

\begin{tabular}{|c|c|c|c|c|c|c|}
\hline \multirow[b]{2}{*}{ Parameters } & \multicolumn{3}{|c|}{ Male groups } & \multicolumn{3}{|c|}{ Female groups } \\
\hline & Control & Nano drug & Free drug & Control & Nano drug & Free drug \\
\hline $\mathrm{WBC}\left(10^{3} / \mu 1\right)$ & $11.60 \pm 0.36$ & $10.20 \pm 0.12$ & $9.60 \pm 0.15^{a}$ & $6.97 \pm 0.62$ & $6.53 \pm 0.18$ & $8.87 \pm 0.41^{a}$ \\
\hline $\operatorname{RBC}\left(10^{6} / \mu 1\right)$ & $7.95 \pm 0.43$ & $7.27 \pm 4.50$ & $4.79 \pm 0.16^{a}$ & $7.15 \pm 0.2$ & $6.49 \pm 0.16$ & $6.1 \pm 0.12^{a}$ \\
\hline $\mathrm{HB}(\mathrm{g} / \mathrm{dl})$ & $12.20 \pm 0.27$ & $12.17 \pm 0.23$ & $11.63 \pm 0.20^{a}$ & $11.33 \pm 0.35$ & $10.90 \pm 0.56$ & $12.07 \pm 0.69$ \\
\hline $\operatorname{HCT}(\%)$ & $37.97 \pm 1.09$ & $36.40 \pm 0.32$ & $36.50 \pm 0.68$ & $37.23 \pm 1.27$ & $33.17 \pm 0.66^{a}$ & $29.50 \pm 0.32^{a}$ \\
\hline MCV (fl) & $50.60 \pm 0.56$ & $49.90 \pm 0.46$ & $50.17 \pm 0.62$ & $50.53 \pm 0.62$ & $51.73 \pm 1.29$ & $51.13 \pm 1.17$ \\
\hline $\mathrm{MCHC}(\%)$ & $32.43 \pm 0.44$ & $33.03 \pm 0.27$ & $36.83 \pm 1.36^{a}$ & $34.07 \pm 0.12$ & $33.60 \pm 1.01$ & $34.77 \pm 0.69$ \\
\hline $\operatorname{PLT}\left(10^{5} / \mu 1\right)$ & $885.33 \pm 8.57$ & $730.33 \pm 34.38$ & $568.00 \pm 12.58^{a}$ & $804.0 \pm 28.01$ & $720.0 \pm 14.42$ & $575.67 \pm 21.18^{a}$ \\
\hline LYM (\%) & $87.93 \pm 0.32$ & $86.57 \pm 2.34$ & $58.27 \pm 1.43^{a}$ & $78.4 \pm 0.84$ & $78.27 \pm 2.21$ & $83.43 \pm 0.93^{a}$ \\
\hline $\mathrm{N}(\%)$ & $7.40 \pm 2.2$ & $8.40 \pm 0.9$ & $10.80 \pm 1.2^{a}$ & $12.40 \pm 2.2$ & $11.40 \pm 1.2$ & $14.00 \pm 0.9^{a}$ \\
\hline
\end{tabular}

The values are expressed as mean \pm SEM of 5 animals. ${ }^{a}$ denotes significant at the level of $p<0.05$.

Table III. Biochemical changes in animals after 28 days of drug administration.

\begin{tabular}{|c|c|c|c|c|c|c|}
\hline \multirow[b]{2}{*}{ Parameters } & \multicolumn{3}{|c|}{ Male groups } & \multicolumn{3}{|c|}{ Female groups } \\
\hline & Control & Nano drug & Free drug & Control & Nano drug & Free drug \\
\hline TB (mg/dl) & $0.85 \pm 0.03$ & $0.95 \pm 0.15$ & $1.25 \pm 0.02$ & $0.97 \pm 0.02$ & $0.93 \pm 0.02$ & $1.10 \pm 0.12$ \\
\hline TP (mg/dl) & $5.43 \pm 0.02$ & $5.56 \pm 0.15$ & $4.07 \pm 0.02^{a}$ & $6.47 \pm 0.34$ & $6.42 \pm 0.28$ & $6.40 \pm 0.24$ \\
\hline AST (IU/L) & $115.5 \pm 9.6$ & $113.2 \pm 10.2$ & $135.5 \pm 9.6^{a}$ & $131.0 \pm 7.2$ & $142.4 \pm 28.2$ & $153.3 \pm 12.3^{a}$ \\
\hline ALT (IU/L) & $56.5 \pm 6.7$ & $57.2 \pm 5.2$ & $60.2 \pm 4.2^{a}$ & $53.7 \pm 4.9$ & $54.1 \pm 4.0$ & $67.2 \pm 4.2^{a}$ \\
\hline ALP (IU/L) & $109.0 \pm 2.52$ & $114.0 \pm 3.46$ & $117.33 \pm 1.76^{a}$ & $160.67 \pm 1.2$ & $165.00 \pm 1.15^{a}$ & $169.00 \pm 1.15^{a}$ \\
\hline CRE (mg/dl) & $0.88 \pm 0.02$ & $1.27 \pm 0.02$ & $1.47 \pm 0.12^{a}$ & $0.96 \pm 0.02$ & $1.20 \pm 0.02$ & $1.67 \pm 0.15^{a}$ \\
\hline
\end{tabular}

The values are expressed as mean \pm SEM of 5 animals. ${ }^{a}$ denotes significant at the level of $p<0.05$.

hepatocytes were damaged in liver (Fig. 4(k)) and tubular degeneration with basophilic foci was found in the kidneys (Fig. 4(1)).

\section{DISCUSSION}

In the recent years, oral delivery of anti cancer drugs encapsulated in polymeric nanoparticles has gained much attention. This is mainly due to the potential of nanoparticles to protect the entrapped drug molecules from the harsh conditions (chemical and enzymatic degradation) of gastro intestinal track. ${ }^{21,22}$ In addition to this, the drug is delivered directly into systemic circulation through the lymphatics circumventing the first pass metabolism, as nanoparticle uptake generally occurs through "M-cells" of the Peyer's patches via receptor mediated endocytosis. ${ }^{5}$

In the present study, we have encapsulated Erlotinib$\mathrm{HCl}$ with PLGA and evaluated the subacute toxicity in rats. Erlotinib- $\mathrm{HCl}$ nanoparticles were prepared by emulsion solvent evaporation method which enabled us to obtain spherical, discrete spheres with a size ranging from 200-300 $\mathrm{nm}$ as revealed by scanning electron microscopy (Fig. 1). After characterizing the nanoparticles, we studied the in vitro release profile (Fig. 2). The in vitro release profile of Erlotinib-PLGA nanoparticles had two distinct phases. The initial burst release phase (for about 4 days) might be due to the dissolution and diffusion of the drug that was poorly entrapped in the polymer matrix. On the other hand, the slower and continuous release after this initial burst can be attributed to the diffusion of the drug localized in the core of the nanoparticles. Such biphasic release profile of the entrapped therapeutic agent from PLGA nanoparticles has been reported before. ${ }^{5,19}$

Inhibitors like Erlotinib that target EGFR, display the broadest spectrum of adverse effects on skin and hair, including folliculitis, paronychia, facial hair growth, facial 

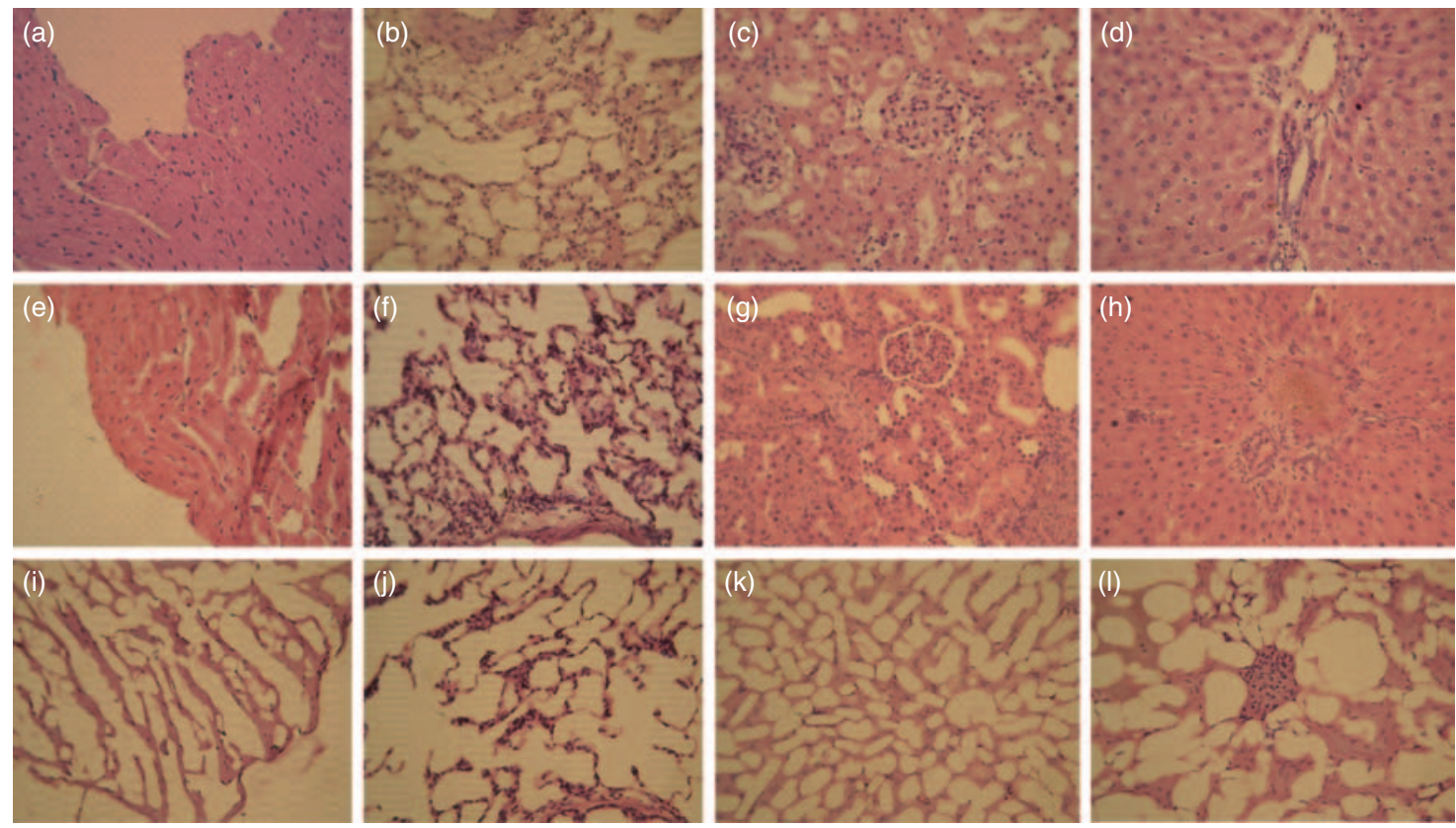

Fig. 4. Photomicrographs of internal organs (heart, lung, liver and kidney) of control and treated animals. (a) Heart of a control animal; (b) Lung of a control animal; (c) Kidney of a control animal; (d) Liver of a control animal; (e) Heart of a nano with Erlotinib-HCl treated animal, (f) Lung of a nano with Erlotinib- $\mathrm{HCl}$ treated animal; (g) Kidney of a nano with Erlotinib- $\mathrm{HCl}$ treated animal and; (h) Liver of a nano with Erlotinib-HCl treated animal. Note the normal architecture in the organs of control and nano Erlotinib- $\mathrm{HCl}$ treated animals. (i) Heart of a free Erlotinib- $\mathrm{HCl}$ treated animal showing cardiac muscle fibres were completely damaged; (j) Lung of a free Erlotinib-HCl treated animal showing normal architecture was lost; (k) Kidney of a free Erlotinib-HCl treated animal showing tubular degeneration with basophilic foci and; (1) Liver of a free Erlotinib-HCl treated animal showing damaged hepatocytes.

erythema, frontal alopecia and induce haematological side effects like anaemia, thrombopenia and neutropenia. ${ }^{23}$ Adverse effects of chemotherapeutic agents are generally accompanied by a loss of body weight or reduced body weight gain, changes in the weight of vital organs, disturbance in haematological, biochemical parameters etc. Hence, rats administered with Erlotinib- $\mathrm{HCl}$ in the nano and free forms were compared in these toxiclological parameters. In our preliminary repeated dose experiments, we found that oral administration of $200 \mathrm{mg} \mathrm{kg}^{-1}$ Erlotinib- $\mathrm{HCl}$ encapsulated in PLGA nanoparticles was well tolerated by rats. Hence, we have chosen this dosage of both nanoencapsulated and free drug for subactue toxicity comparison studies.

The data obtained in the present study clearly indicate that administration of $200 \mathrm{mg} \mathrm{kg}{ }^{-1}$ Erlotinib- $\mathrm{HCl}$ has induced systemic toxicity in rats. For e.g., haematological analyses have shown that these animals have developed anaemia and reduced immunity. It has already been reported that Erlotinib causes a reduction in RBC with a compensatory increase in reticulocytes, white blood cells and primarily neutrophils in rats. ${ }^{24}$ Another important adverse effect of chemotherapy is the tissue damage or necrosis of organs which is generally accompanied by increase in the levels of several non-functional plasma enzymes such as AST, ALT and ALP. These enzymes are among several other enzymes used for the diagnostic purpose, ${ }^{25}$ since these enzymes present in liver and heart cells and are normally released into blood only when these organs are damaged or undergo tissue necrosis. In the present study also levels of these enzymes have significantly increased in the plasma of rats administered with free Erlotinib- $\mathrm{HCl}$ indicating severe damage to liver and/or heart tissue.

The toxicological changes in the free drug treated animals were further apparent in the histopathological studies. We observed tissue damage or necrosis in all the tested internal organs, namely heart, lung, kidney and liver. As seen in the photomicrographs, cardiac muscle fibres were completely damaged in the heart (Fig. 4(i)), normal architecture was lost in lung (Fig. 4(j)), tubular degeneration with basophilic foci was found in the kidneys (Fig. 4(k)) and hepatocytes were damaged in liver (Fig. 4(1)).

Although toxicity symptoms were apparent in animals administered with free Erlotinib-HCl, there was no sign of toxicity observed in animals administered with nanoencapsulated form of the drug of the same dose. All the haematological and biochemical parameters tested were normal and histopathological studies confirmed normal architecture of the tested internal organs. Hence, our study clearly show that nanoencapsulation with PLGA protects the animals from Erlotinib- $\mathrm{HCl}$ induced toxicity at a concentration which is otherwise not tolerated by these animals in repeated doses. Nanoparticles have made a tremendous impact in the treatment of various types of cancer, as evidenced by the numerous nanoparticle-based drugs 
and delivery systems that are in clinical use. ${ }^{26}$ Several anti-cancer drugs including paclitaxel, ${ }^{27,}{ }^{28}$ doxorubicin, ${ }^{29}$ 5 -fluorouracil ${ }^{30}$ and dexamethasone ${ }^{31}$ have been successfully formulated using nanomaterials.

Although many reports are available on nanoformulations of anticancer drugs, in vivo toxicity studies are relatively scarce. In this context, cyclosporine-induced nephrotoxicity could be significantly reduced by nanoencapsulation of this drug with PLGA. ${ }^{32}$ Similarly, cardiac toxicity of doxorubicin was dramatically reduced by PLGA encapsulation. ${ }^{15,33}$ Moreover, estradiol loaded PLGA nanoparticles was effective in improving the oral bioavailability and decreasing the dosing frequency, thereby minimizing the dose dependent adverse effects in male Sprague Dawley (SD) rats. ${ }^{34}$ All these reports support our observation that PLGA nanoencapsulation can significantly reduce drug-induced toxicity. The reduced toxicity of Erlotinib-HCl loaded in PLGA nanoparticles may be attributed to the pharmacokinetics of the nanoformulation, which warrants further investigation.

\section{CONCLUSION}

The results of this study clearly indicate that there is a great potential for nanoparticulate delivery of Erlotinib- $\mathrm{HCl}$ in minimizing drug-induced toxicity. However, extensive studies in terms of chronic toxicity, pharmacokinetic and pharmacodynamic are needed before establishing nanoparticle-mediated delivery of this drug.

\section{References and Notes}

1. S. S. Sridhar, L. Seymour, and F. A. Shepherd, Inhibitors of epidermal-growth-factor receptors: A review of clinical research with a focus on nonsmall-cell lung cancer. Lancet Oncol. 4, 397 (2003).

2. Y. Yarden and M. X. Sliwkowski, Untangling the ErbB signaling network. Nat. Rev. Mol. Cell Biol. 2, 127 (2001).

3. A. Sandler and R. Herbst, Combining targeted agents: Blocking the epidermal growth factor and vascular endothelial growth factor pathways. Clin. Cancer Res. 12, 4421 (2006).

4. R. Pérez-Soler, A. Chachoua, L. A. Hammond, E. K. Rowinsky, and M. Huberman, Determinants of tumor response and survival with erlotinib in patients with non-small-cell lung cancer. J. Clin. Oncol. 22, 3238 (2004).

5. D. K. Sahana, G. Mittal, V. Bhardwaj, and M. N. V. Ravikumar, PLGA nanoparticles for oral delivery of hydrophobic drugs: Influence of organic solvent on nanoparticle formation and release behaviour in vitro and in vivo using estradiol as a model drug. J. Pharm. Sci. 97, 1530 (2008).

6. K. S. Kumar, V. Selvaraj, and M. Alagar, Synthesis of PETPLA/Drug nanoparticles and their effect with gold nanoparticles for controlled drug release in cancer chemotherapy. Res. Lett. Nanotechnol. 2008, 4 (2008).

7. S. D. Perrault, C. Walkey, T. Jennings, H. C. Fischer, and W. C. Chan, Mediating tumor targeting efficiency of nanoparticles through design. Nano Lett. 9, 1909 (2009).

8. G. S. Kwon and T. Okano, Polymeric micelles as new drug carriers. Adv. Drug Deliv. Rev. 16, 107 (1996).
9. H. S. Yoo, J. E. Oh, K. H. Lee, and T. G. Park, Biodegradable nanoparticles containing doxorubicin-PLGA conjugates for sustained release. Pharm. Res. 16, 1114 (1999).

10. E. Allemann, R. Gurny, and J. C. Leroux, Boidegradable nanoparticles of poly(lactic-co-glycolic acid) for parenteral administration, Pharmaceutical Dosage Forms: Disperse Systems, edited by H. A. Liberman, M. M. Reiger, and G. S. Banker, Marcel Dekker, New York (1996), pp. 163-194.

11. K. S. Soppimath and T. M. Aminabhavi, Ethyl acetate as a dispersing solvent in the production of poly(D,L-lactideco-glycolide) microspheres: Effect of process parameters and polymer type. J. Microencapsulat. 19, 281 (2002).

12. M. M. Arnold, E. M. Gorman, L. J. Schieber, E. J. Munson, and C. Berland, NanoCipro encapsulation in monodisperse large porous PLGA microparticles. J. Control. Release 16, 100 (2007).

13. R. A. Jain, The manufacturing techniques of various drug loaded biodegradable poly(lactide-co-glycolide) (PLGA) devices. Biomaterials 21, 2475 (2000).

14. I. Bala, S. Hariharan, and M. N. V. R. Kumar, PLGA nanoparticles in drug delivery: The state of the art. Crit. Rev. Ther. Drug Carrier Syst. 21, 387 (2004).

15. R. Lin, L. Shi Nq, and C. H. Wang, In vitro study of anticancer drug doxorubicin in PLGA-based microparticles. Biomaterials 26, 4476 (2005).

16. O. C. Farokhzad, J. Cheng, B. A. Teply, I. Sherifi, S. Jon, P. W. Kantoff, J. P. Richie, and R. Langer, Targeted nanoparticle-aptamer bioconjugates for cancer chemotherapy in vivo. Proc. Natl. Acad. Sci. USA 103, 6315 (2006).

17. A. Budhian, S. J. Siegel, and K. I. Winey, Haloperidol-loaded PLGA nanoparticles: Systematic study of particle size and drug content. Int. J. Pharm. 336, 367 (2007).

18. S. K. Jain, Y. Gupta, A. Jain, A. R. Saxena, P. Khare, and A. Jain, Mannosylated gelatin nanoparticles bearing an anti-HIV drug didanosine for site-specific delivery. Nanomedicine 4, 41 (2008).

19. F. Danhier, N. Lecouturier, B. Vroman, C. Jérôme, J. MarchandBrynaert, O. Feron, and V. Préat, Paclitaxel-loaded PEGylated PLGA-based nanoparticles: In vitro and in vivo evaluation. J. Control. Release 133, 11 (2009).

20. K. Park, G. Y. Lee, Y. S. Kim, M. Yu, R. W. Park, I. S. Kim, S. Y. Kim, and Y. Byun, Heparin-deoxycholic acid chemical conjugate as an anticancer drug carrier and its antitumor activity. J. Control. Release 114, 300 (2006).

21. V. Bhardwaj, S. Hariharan, I. Bala, A. Lamprecht, N. Kumar, R. Panchagnula, and M. N. V. Ravi Kumar, Pharmaceutical aspects of polymeric nanoparticles for oral delivery. J. Biomed. Nanotechnol. 1, 235 (2005).

22. A. T. Florence, Nanoparticle uptake by the oral route: Fulfilling its potential? Drug Discovery Today Technol. 2, 75 (2005).

23. J. T. Hartmann, M. Haap, H. G. Kopp, and H. P. Lipp, Tyrosine kinase inhibitors-A review on pharmacology, metabolism and side effects. Curr. Drug Metab. 10, 470 (2009).

24. EMEA, 2005. http://www.emea.europa.eu/humandocs/PDFs/EPAR/ tarceva/061805en6.pdf (accessed on 16 Aug 2009).

25. A. O. Amakiri, O. J. Owen, E. M. Ngodigha, and D. O. Jack, Effect of refined petroleum product (kerosene) flame and fumes on serum enzyme characteristics of broiler chickens. Int. J. Poult. Sci. 7, 1039 (2008).

26. S. K. Murthy, Nanoparticles in modern medicine: State of the art and future challenges. Int. J. Nanomedicine 2, 129 (2007).

27. C. Fonseca, S. Simoes, and R. Gaspar, Paclitaxel-loaded PLGA nanoparticles: Preparation, physicochemical characterization and in vitro anti-tumoral activity. J. Control. Release 83, 273 (2002).

28. J. M. Koziara, T. R. Whisman, M. T. Tseng, and R. J. Mumper, In-vivo efficacy of novel paclitaxel nanoparticles in paclitaxelresistant human colorectal tumors. J. Control. Release 112, 312 (2006). 
29. H. S. Yoo, K. H. Lee, J. E. Oh, and T. G. Park, In vitro and in vivo anti-tumor activities of nanoparticles based on doxorubicin-PLGA conjugates. J. Control. Release 68, 419 (2000).

30. D. Bhadra, S. Bhadra, S. Jain, and N. K. Jain, A PEGylated dendritic nanoparticulate carrier of fluorouracil. Int. J. Pharm. 257, 111 (2003).

31. J. Panyam and V. Labhasetwar, Sustained cytoplasmic delivery of drugs with intracellular receptors using biodegradable nanoparticles. Mol. Pharm. 1, 77 (2004).

32. J. L. Italia, D. K. Bhatt, V. Bhardwaj, K. Tikoo, and M. N. V. Ravi Kumar, PLGA nanoparticles for oral delivery of cyclosporine: Nephrotoxicity and pharmacokinetic studies in comparison to Sandimmune Neoral. J. Control. Release 119, 197 (2007).

33. D. R. Kalaria, G. Sharma, V. Beniwal, and M. N. V. Ravi Kumar, Design of biodegradable nanoparticles for oral delivery of doxorubicin: In vivo pharmacokinetics and toxicity studies in rats. Pharmaceutical Res. 26, 492 (2008).

34. G. Mittal, D. K. Sahana, V. Bhardwaj, and M. N. V. Ravi Kumar, Estradiol loaded PLGA nanoparticles for oral administration: Effect of polymer molecular weight and copolymer composition on release behavior in vitro and in vivo. J. Control. Release 119, 77 (2007).

Received: xx Xxxx xxxx. Revised/Accepted: xx Xxxx xxxx. 